\title{
研究通讯
}

\section{Fuzzy 分块矩阵的一个定理}

基于 Fuzzy 矩阵的可乘性分块:

$$
\begin{aligned}
& F=\left[\begin{array}{ll}
F_{p p} & F_{p q} \\
F_{q p} & F_{q q}
\end{array}\right]_{p+q=n}, \\
& G=\left[\begin{array}{ll}
G_{p p} & G_{p q} \\
G_{q p} & G_{q q}
\end{array}\right]_{p+q=n},
\end{aligned}
$$

作者定义了 Fuzzy 分块矩阵的乘法 $\odot$ : $F \bigodot G$

$$
\triangleq\left[\begin{array}{ll}
\left(F_{p p} \circ G_{p p}\right) \cup\left(F_{p q} \circ G_{q p}\right) & \left(F_{p p} \circ G_{p q}\right) \cup\left(F_{p q} \circ G_{q q}\right) \\
\left(F_{q p} \circ G_{p p}\right) \cup\left(F_{q q} \circ G_{q p}\right) & \left(F_{q p} \circ G_{p q}\right) \cup\left(F_{q q} \circ G_{q q}\right)
\end{array}\right],
$$

式 (2) 中的符号 0 是指 Fuzzy 矩阵的乘法运算(或 称合成运算)。

接着,作者证明了关于 Fuzzy 分块矩阵乘法的 一个定理:

定理 1 Fuzzy 矩阵 $F$ 对 $G$ 的分块矩阵乘法 $F \odot G$ 等效于 $F$ 对 $G$ 的乘法 $F \circ G$, 即
$F \odot G \equiv F \circ G$

(3)

或写成

$$
\begin{gathered}
{\left[\begin{array}{ll}
F_{p p} & F_{p q} \\
F_{q p} & F_{q q}
\end{array}\right] \odot\left[\begin{array}{ll}
G_{p q} & G_{p q} \\
G_{q,} & G_{q q}
\end{array}\right]_{p+q=n}} \\
\equiv\left(f_{i k}\right)_{n \times l} \circ\left(g_{k j}\right)_{i \times n} .
\end{gathered}
$$

这个定理应用于下面的特例是格外有价值的：

若

$$
F=G=\left[\begin{array}{ll}
F_{p l} & O_{p q} \\
O_{q L} & F_{q q}
\end{array}\right]_{p+q=n},
$$

即得到

$$
F^{2}=F \odot F=\left[\begin{array}{cc}
\left(F_{p p}\right)^{2} & O_{p q} \\
O_{q p} & \left(F_{q q}\right)^{2}
\end{array}\right],
$$

式中的 $O_{p \times q}, O_{q \times p}$ 分别为 $p \times q 、 q \times p$ 的零矩阵.

涂象初涂承媛

(北京工业大学三系)

\section{循 环 差 集 的 额 外 乘 数*}

H. B. Mann 首先研究了 $(v, k, \lambda)$ 循环差集的额 外乘数一一非 $n=k-\lambda$ 因数的乘数, 并得到了整 数 2 不是任一非平凡循环差集的额外乘数这一有趣 的结果. 本文证明了 Mann (元 Some Theorems on Difference Sets, Canadian J. Math, 1952, 222226) 的一个定理的逆,得到

定理 1 索数 $p$ 是一 $(v, k, \lambda)$ 循环差集 $D$ 的 额外乘数当且仅当

$$
\theta^{p-1}(x) \equiv x^{s}, \quad \bmod d\left(p, x^{v}-1\right)
$$

或

$$
\theta^{p-1}(x) \equiv x^{s}-T(x), \bmod d\left(p, x^{v}-1\right)
$$

对某一整数成立，且 (1) 式成立时 $p \nmid k ，(2)$ 式成 立时 $p \mid k$.

* 国家教委搏士点基金资助的课题.

第 18 期
我们还证明了

定理 2 一奇素数 $p$ 是一 $(v, k, \lambda)$ 循环差集 的额外乘数当且仅当

$$
\begin{gathered}
n^{\frac{p-1}{2}} \theta^{\frac{p-1}{2}}(x)+\frac{1}{\nu} k^{\frac{p-1}{2}}\left(1-n^{\frac{p-1}{2}}\right) T(x) \\
\equiv x^{s} \theta^{\frac{p-1}{2}}\left(x^{-1}\right) \bmod d\left(p, x^{\nu}-1\right)
\end{gathered}
$$

对某一整数 $s$ 成立.

上面的 (1)，(2)、(3) 式中的 $\theta(x)$ 是 $D$ 的 Hall 多项式, 而

$T(x) \equiv 1+x+\cdots+x^{v-1} \bmod \left(x^{v}-1\right)$.

利用定理 2 , 我们证明了

定理 3 整数 3 不是任一非平凡循环差集的 额外乘数。也就是说, 如果 3 是一非平凡的 $(\nu, k, \lambda)$ 循环差集的乘数, 则 $3 \mid n=k-\lambda$.

科 学 通 报 
定理 4 整数 5 不是任一非平风平面循环差 集的额外乘数。也就是说, 如 5 是一非平凡的 $(v, k$, 1)循环差集的乘数, 则 $5 \mid n=k-1$.

结合乘数定理, 定理 4 是说, 5 是非平凡平面循 环差集的乘数当且仅当 $5 \mid n=k-1$. 加上 Mann 已证明的结果,我们有

推论 设 $D$ 是非平凡的 $(v, k, 1)$ 循环差集; 那 么:

$1^{\circ} 2$ 是 $D$ 的乘数当且仅当 $2 \nmid v$ 且

$$
2 \mid x=k-1 \text {. }
$$

$2^{\circ} 3$ 是 $D$ 的乘数当且仅当 $3 \nmid v$ 且

$$
3 \mid n=k-1 \text {. }
$$

$3^{\circ} 5$ 是 $D$ 的乘数当且仅当 $5 \nmid 0$ 且

$$
5 ! n=k-1 \text {. }
$$

致谢：本文作者衷心感谢魏万迪老师的热情鼓 励和细心指导。

吴晓红

(四川大学数学系, 成都)

\section{随机规划中关于正态分布的若千凸性命题}

随机规划中有一类机会约束规划问题, 其一般 形式为

$$
X\langle\alpha\rangle \triangleq\{x \mid p(\omega \mid A(\omega) x \geqslant b(\omega)) \geqslant \alpha, x \in X\}
$$

或者

$$
X_{i}\left(\alpha_{i}\right) \triangleq\left\{x \mid p\left(\omega \mid A_{i}(\omega) x \geqslant b_{i}(\omega)\right) \geqslant \alpha_{i}, x \in X\right\}
$$

是否凸集.

颜铁成讨论了 $A(\omega)$ 的所有元素为独立的正态 分布随机变量而 $b(\omega)$ 固定时的凸性命题。本文讨 论 $A(\omega)$ 的若干列元素为独立的标准正态分布随机 变量，而其他元素固定时的凸性命题，得到如下结 杲:

定理 1 如 $a_{i j}(\omega) \sim N(0,1)(j=1, \cdots, N$; $1 \leqslant N \leqslant n)$ 及 $a_{i j}(\omega)(j=1, \cdots, N ; 1 \leqslant N \leqslant n)$ 相 互独立而其他元素固定, 则

(i) 如果

$$
x \subset\left\{\left(x_{1}, \cdots, x_{n}\right) \mid \sum_{K=N+1}^{n} a_{i K} x_{K} \geqslant b_{i}\right\},
$$

那末 $X_{i}\left(\alpha_{i}\right)$ 为蓝;

(ii) 如果

$$
X \subset\left\{\left(x_{1}, \cdots, x_{n}\right) \mid \sum_{K=N+1}^{n} a_{i K} x_{K}<b_{i}\right\}, 0<\alpha_{i}<1,
$$

那末 $X_{i}\left(\alpha_{i}\right)$ 为非凸集.

定理 2 如 $a_{i j}(\omega) \sim N(0,1) \quad(i=1, \cdots, m$; $1=1, \cdots, N ; 1 \leqslant N \leqslant n), a_{i j}(\omega)(i=1, \cdots, m$; $1=1, \cdots, N ; 1 \leqslant N \leqslant n)$ 相互独立而其他元素固
定, 则

(i) 如果

$$
X \subset\left\{\left(x_{1}, \cdots, x_{n}\right) \mid \sum_{K=N+1}^{n} a_{i K} x_{K} \geqslant b_{i}, z=1, \cdots, m\right\} \text {. }
$$

那末 $X(\alpha)$ 为凸集;

(ii) 如果存在 $i_{0} \in\{1, \cdots, m\}$ ，使

$$
\begin{aligned}
& \sum_{K=N+1}^{n} a_{i_{0}} K^{x_{K}}<b_{i_{0}}, \\
& x \subset\left\{\left(x_{1}, \cdots, x_{n}\right) \mid \sum_{K=N+1}^{n} a_{i_{0}} K^{\left.x_{K}<b_{i_{0}}\right\},}\right. \\
& 0<\alpha<1,
\end{aligned}
$$

那末 $X(\alpha)$ 为非凸集。

定理 3 如 $a_{i j}(\omega) \sim N(0,1) \quad(j=1, \cdots, n)$ ， $b_{i}(\omega) \sim N(0,1)$ 及 $a_{i j}(\omega)(j=1, \cdots, n), b_{i}(\omega)$ 相 互独立,那末 $X(\alpha)$ 为凸集。

定理 4 如 $a_{i j}(\omega) \sim N(0,1), b_{i}(\omega) \sim N(0$ ， 1) $(i=1, \cdots, m ; j=1, \cdots, n)$ 及 $a_{i j}(\omega), b_{i}(\omega)(i=$ $1, \cdots, m ; 1=1, \cdots, n)$ 相互独立, 则 $X(\alpha)$ 是凸集。

显然, 当 $N=n$ 时, 定理 2 给出了颜铁成所指出 的部分结果.

关于 $X(\alpha)=\{x \mid p(\omega \mid A(\omega) x \leqslant b(\omega)) \geqslant \alpha\}$ 的 凸性, 我们也有平行的结果。

雷忠学

(苏州铁道师范学院数学系)

\section{添加 $1.5 \% \mathrm{~W}$ 对 $\mathrm{Fe}-\mathrm{Cr}-\mathrm{Co}-\mathrm{V}-\mathrm{Ti}$ 永磁体的影响}

$\mathrm{Fe}-\mathrm{Cr}-\mathrm{Co}-\mathrm{V}-\mathrm{Ti}$ 永磁体的特点, 是不存在有害 相, 因为 $\mathrm{V}$ 具有扩大 $\alpha$ 相的作用. 初期, 需用的 $\mathrm{V}$ 量
为 3 5\% (重量\%)(金子秀夫等, 1976、1878). 其 $B$, 值和 $(B H)_{\max }$ 值近于半柱状晶的 AINiCO5 永磁 